\title{
The Portuguese Independent Living Support Model (MAVI)
}

\author{
José Manuel Silva* \\ Santa Maria Health School, Portugal
}

*Corresponding author: José Manuel Silva, Dean of Santa Maria Health School, Portugal.
Received Date: January 31, 2020

Published Date: March 18, 2020

\section{Opinion}

Some civic associations have sought to implement replicas of integrable solutions in Portugal to the model of the Movement of Independent Living. Those have been limited experiences with little expression although they have taken a pioneering path that has now found acceptance in public policies.

In 2017, the Portuguese Government launched the Independent Life Support Model (MAVI) for people with severe physical or mental limitations, providing for the existence of pilot projects for the period 2017-2020. The model is based on three dimensions/ agents

- Person with a disability who benefits from Personal Assistance.

- Personal assistant.

- Support Center for Independent Living (CAVI), an organization which organizes support and manages the funds at its disposal.

All people with disabilities who need support to continue their lives independently can be beneficiaries of the project, namely if they have a disability or inability over $60 \%$ or in case they have intellectual limitations, autism spectrum disorders or mental illness, regardless of their degree of incapacity. They must register with the CAVI, entities that are responsible for sorting candidates and giving them the necessary and possible support according to the available funding and the existing conditions.

Personal assistance is a specialized service to support independent living that allows beneficiaries to carry out activities | which, due to the limitations resulting from their interaction with environmental conditions, they cannot perform on their own. The support can be translated into personal assistance in hygiene activities, food, health maintenance and personal care, housework, work context, travel, participation in civic activities, mediation of communication, frequency of vocational training or higher education and research, culture, leisure and sport. The assistance can range from a reduced number of hours to permanent monitoring, according to the needs of the beneficiary and the resources available.

Every beneficiary has an individualized personal assistance plan, a program document conceived with their own participation or, in case that is not possible, with their legal representative. It is adjusted to their needs and decisions and it should correspond to their life project, their vision of the future, motivations and desires.

Personal assistants are people who provide support to beneficiaries and whose basic requirements for recruitment are adulthood and basic education, and they must also have a set of requirements ranging from reputability to physical strength and others that guarantee the minimum desirable conditions for those who are meant to assist people with special needs in daily life activities.

They also need to attend a 50-hour training program and if they are selected, they are part of a pool of professionals who will be called when needed. They can work part-time or full-time, depending on their availability and on the individualized support programs in which they participate. The CAVI are created on the initiative of private entities that apply for the program, normally 
already active in the area, and they are selected by the National Institute for Rehabilitation (INR), the entity that manages the overall project. The CAVI manage the financial support provided by the State and they are responsible for providing the personal assistance service to the beneficiaries who choose them, hiring personal assistants and managing the entire process locally.

This pilot program represents a step forward in ensuring, in Portugal, a set of minimum support for people in need of personal assistance in activities which they are unable to carry out on their own, providing them with specific support, greater autonomy and decision-making capacity. As far as social rights are concerned, it is an important milestone as it recognizes that independent living is a right every citizen should have, and it acknowledges that the State is responsible for financing and ensuring the necessary support to provide these people with the best possible standards of independence, despite the limitations resulting from specific personal situations.

Although the program may be criticized since the funding is not allocated directly to the beneficiaries, but to the CAVI, who then hire personal assistants and manage the program locally, the country's situation and the most common organizational characteristics must be taken into account, considering the process as an experience to be evaluated, preparing future interventions that may eventually be more adapted to the interests of the beneficiaries, the characteristics of the CAVI and the financial availability of the Portuguese State.

As a scientific contribution to the partial evaluation of the program, a research-action-assessment project called ICAVI, was created and is currently being developed which aims to monitor and assess the form of its implementation in a group of CAVI, under the responsibility of researchers from universities, higher education institutions, CAVI technicians and the Center for Research in Technology and Health Services (CINTESIS).

\section{Acknowledgement}

None.

\section{Conflict of Interest.}

No conflict of interest. 